\title{
A Study of Localization Accuracy Using Multiple Frequencies and Powers
}

\author{
Xiuyuan Zheng, Hongbo Liu, Jie Yang, Yingying Chen, Richard P. Martin, and Xiaoyan Li
}

\begin{abstract}
Wireless localization using the received signal strength (RSS) can have tremendous savings over using specialized positioning infrastructures. In this work, we explore improving RSS localization performance in multipath environments by varying the transmitter's signal power and frequency. We first derive and analyze the Cramér-Rao Lower Bound (CRLB) of RSS-based localization based on the frequency dependent path loss propagation model that considers the transmitter's signal power and frequency. The derived CRLB shows the feasibility of improving localization performance by applying frequency and power level selection for RSS-based localization. Using this analysis, we develop two new selection metrics based on the observed standard deviations of RSS as well as residuals. We then show a set of selection methods that attempt to select the combinations of power and frequencies which minimize the localization error in a representative class of localization algorithms. Our simulation results confirm the proposed selection methods can improve the localization accuracy under CRLB. Additionally, using active RFID tags, we experimentally characterize the effect of using multiple signal powers and frequencies on a wide spectrum of RSS-based algorithms. We found that the performance of all the algorithms improves when leveraging on multiple power levels and frequencies, although different algorithms present different sensitivity in terms of localization accuracy under different selection methods.
\end{abstract}

Index Terms-Wireless localization, received signal strength, multiple frequency, multiple power level

\section{INTRODUCTION}

$\mathrm{U}$ SING wireless devices to provide location is an emerging area that will impact a diverse set of applications including those in asset tracking, workflow management, geographic routing, and physical security. Given that wireless devices are carried by many people and objects, and all modern radio chipsets include the hardware necessary to measure the received signal strength (RSS) of transmitted packets, there is a tremendous cost and deployment advantage to re-using the existing RSS infrastructure of the communication network for localization.

The basic strategy of most RSS-based localization follows a typical pattern: a transmitter or receiver, called the device, measures the RSS to a number of landmarks with known positions. The resulting collection of RSS values, or fingerprint, is then used to position the device. A wide range of algorithmic strategies have been tried, ranging from minimization of least squares methods to machine learning approaches.

- X. Zheng, Y. Chen are with the Department of Electrical and Computer Engineering, Stevens Institute of Technology, Hoboken, NJ 07030 USA. E-mail: \{xzheng1, hliu3, yingying.chen\}@stevens.edu.

- H. Liu, is with the Department of Computer Information Technology, Indiana University-Purdue University Indianapolis, Indianapolis, IN 46202 USA.Email: hl45@iupui.edu.

- J. Yang is with the Department of Computer Science and Engineering, Oakland University, Rochester, MI 48309 USA. E-mail: yang@oakland.edu.

- R.P. Martin is with the Department of CS, Rutgers University, Piscataway, NJ 08854 USA. E-mail: rmartin@cs.rutgers.edu.

- X. Li is with the Department of CS, Lafayette College, Easton, PA 18042 USA.E-mail: lix@lafayette.edu.

- The preliminary results have been published in IEEE MASS 2010 [19].

Manuscript received 17 Apr. 2013; revised 19 Sept. 2013; accepted 23 Oct. 2013. Date of publication 5 Nov. 2013; date of current version 16 July 2014. Recommended for acceptance by J. Cao.

For information on obtaining reprints of this article, please send e-mail to: reprints@ieee.org, and reference the Digital Object Identifier below.

Digital Object Identifier no. 10.1109/TPDS.2013.281
However, multipath effects are key challenge to wireless localization using RSS. These effects include shadowing, i.e., blocking a signal, reflection, i.e., waves bouncing off an object, diffraction, i.e., waves spreading in response to obstacles, and refraction, i.e., waves bending as they pass through different mediums. These effects impact the RSS, and properly accounting for all of them in complex indoor environments is a difficult task. Typically, the average localization errors are around $10 \mathrm{ft}$ and maximum errors are often $30 \mathrm{ft}$ or more.

In this paper we explore one approach of improving RSS localization performance in face of multipath effects. Our strategy is for the transmitter to vary its signal frequency and power level, thus generating multiple RSS values between the device and each landmark. The resulting distinct RSS values for each frequency and power level combination create a larger fingerprint than is typical with a single frequency and power level. We call each frequency and power level combination a dimension of the fingerprint. We then apply a selection algorithm that attempts to create a fingerprint that minimizes the localization error.

We first analyze the performance of RSS-based localization by deriving the Cramér-Rao Lower Bound (CRLB) based on the frequency dependent path loss propagation model that considers the transmitter's signal power and frequency. Our analysis forms a general view of localization accuracy by selecting RSS measurements from multiple frequencies and power levels. The analysis shows the feasibility of improving localization performance by applying selection on RSS measurements from multiple frequencies and power levels.

We use two metrics to help select the higher-quality signal strength fingerprint based on the theoretical analysis: deviation of RSS readings and residual. We further develop three selection strategies: 1) Whole Dimension, whereby all 
dimensions of frequency and power level are used for localization; 2) Matching Characteristic, whereby a fingerprint is constructed with all landmarks using the same frequency and power level combination; and 3) Constrained Landmark, whereby all landmarks are enforced to be used, but allowing any dimension to be chosen for a landmark.

We apply our approach to a broad class of localization algorithms with a range of mathematical foundations, from lateration based, to fingerprint matching, to probabilistic based, and Bayesian Networks. Our first step analysis centers on the Linear Least Squares (LLS) algorithm; we theoretically show that selection should improve performance, and the selection should take the dimensions with the minimum variance within a dimension. We next evaluate our proposed selection strategies through a simulated indoor environment by comparing the theoretical CRLB and the RMS (Root Mean Square) error. We observe that the simulation results of localization algorithms are bounded by the theoretical CRLB values.

Furthermore, by leveraging a real localization testbed, we programmed RFID transmitters with varying frequency and power level. We are able to vary the frequency at two levels, $400 \mathrm{MHz}$ and $900 \mathrm{MHz}$ as well as simultaneously vary the power by 3 levels, 10, 5, and $0 \mathrm{dBm}$, resulting in 6 additional dimensions of RSS between a device and a landmark. We then use a trace-driven approach to evaluate the impact of our selection strategies on this broad class of localization algorithms.

We find that using multiple frequencies and power levels improved the performance of all the algorithms. An interesting effect common across all the algorithms is that median errors are substantially reduced. Examining a cumulative distribution function (CDF) of localization errors, the median errors improve over 40 percent for all the algorithms using multiple frequencies and power levels, whereas the improvement of 90th percentile errors varies from 32 percent to 58 percent depending on the algorithm. We further find that different algorithms react differently to the selection strategies. Selection is necessary to improve performance and reduce computational cost compared to using Whole Dimension selection, although each algorithm required a slightly different selection strategy. We summarize our main contributions as follows:

- We theoretically analyze the performance of RSSbased localization by deriving the Cramér-Rao Lower Bound (CRLB) based on the frequency dependent path loss propagation model that considers the transmitter's signal power and frequency. The analysis shows the feasibility of improving localization accuracy by applying frequency and power level selection to RSS-based localization.

- We design two selection metrics: the deviation of RSS readings and the residual to characterize the impact of multiple frequencies and power levels on wireless localization. We design three selection strategies: Whole Dimension, Matching Characteristic, and Constrained Landmark to help select the higher-quality fingerprint.

- We study a broad class of localization algorithms including lateration based, fingerprint matching, probabilistic based, and Bayesian Networks algorithms. The results show that our approach is generic enough to apply to any RSS-based localization algorithms.

- We conduct extensive simulation and real experiments to validate our approaches. Both simulation and experimental results are consistent with our theoretical analysis, and show that using multiple frequencies and power levels can improve the performance of all the algorithms under study.

The remainder of our paper is organized as follows. We first derive the CRLB of localization using RSS measurements in Section 2 of both the main paper and the supplementary file which is available in the Computer Society Digital Library at http://doi.ieeecomputersociety. org $/ 10.1109 / 281$. We then develop our selection metrics and describe the broad class of localization algorithms in Section 3 of both the main paper and the supplementary file available online. Next, we present our selection strategies of multiple frequencies and power levels for localization and perform simulation study on different localization algorithms to compare with the theoretical bounds in Section 4 of the main paper. In Section 5 of the main paper and Section 4 of the supplementary file available online, we describe our testbed and present experimental results. Finally, Section 6 concludes our work. The related work is discussed in Section 1 of the supplementary file available online.

\section{Theoretical analysis on Localization Performance}

In this section, we study the performance of RSS-based localization by deriving the Cramér-Rao Lower Bound (CRLB) based on the frequency dependent path loss propagation model. We first introduce a frequency dependent path loss propagation model that considers the transmitter's signal power and frequency. Then, the CRLB of RSS-based localization is derived based on the frequency dependent path loss propagation model. We investigate the feasibility of improving localization performance by applying frequency and power level selection for RSS-based localization. More details of the derivation and discussions are presented in Section 2 of the supplementary file available online.

\subsection{Frequency and Power Dependent Path Loss Propagation Model}

The path loss propagation model [16] can be described as, $P(d)=P\left(d_{0}\right)-10 \beta \log \left(\frac{d}{d_{0}}\right)+X_{\sigma}$, where $\beta$ is the path loss exponent, $X_{\sigma}$ represents the shadowing effect parameter with variance $\sigma^{2}\left(X_{\sigma} \sim N\left(0, \sigma^{2}\right)\right)$. $P(d)$ is the received power of a wireless device at a certain location with distance $d$ to the landmark. $P\left(d_{0}\right)$ can be considered as a power loss in free space, where $d_{0}$ is usually defined as 1 meter.

The transmission power $P\left(d_{0}\right)$ usually depends on the frequency used for transmitting packets. Therefore, $P\left(d_{0}\right)$ can be written as a transmission frequency dependent parameter [1], [7], $P\left(d_{0}\right)=\left(20 \log (f)_{(\mathrm{MHz})}-\tau\right)$, where $\tau$ is a constant environmental parameter (empirically set to 27 . 56 in [1]). 
And the path loss exponent $\beta$ depicts the rate of RSS varying with distance, which tends to increase when the device operates on higher transmission frequency [1], [10]. This indicates that path loss exponent is proportional to the transmission frequency: $\beta \propto f_{(\mathrm{MHz})}$. Furthermore, a recent work proposes a calibration method to tune the parameters of the propagation model including the path loss exponent $\beta$ to improve the localization performance [3].

Finally, the shadowing, $X_{\sigma}$, is the effect that the received signal power fluctuates due to obstacles in the propagation paths. It has been shown experimentally that the shadowing is dependent on the transmitting frequency and power level [11].

Based on the path loss propagation model, the joint probability density function of the RSS measurements collected from $N(i=1,2, \ldots, N)$ landmarks independently, can be written as:

$$
f_{\theta}(P)=\prod_{i=1}^{N} \frac{10}{\log 10 \sqrt{2 \pi} \sigma_{i}} \frac{1}{P_{i}} \exp \left[-\frac{\rho_{i}}{8}\left(\log \frac{d_{i}^{2}}{\hat{d}_{i}^{2}}\right)^{2}\right],
$$

where $P=\left[P_{1} \ldots P_{N}\right]$ is a vector of the received power of $N$ landmarks, $\rho_{i}=\left(\frac{10 \beta_{i}}{\sigma_{i} \log 10}\right)^{2}$ and $\hat{d}_{i}=d_{0}\left(\frac{P\left(d_{0}\right)}{P_{i}}\right)^{\frac{1}{\beta_{i}}}$ is the estimate of distance $d_{i}$ to the $i$ th landmark. And $\theta=(x, y)$ is the unknown location of the wireless device to be estimated from RSS measurements. Note that the parameter $\rho_{i}$ containing the path loss exponent $\beta_{i}$ and the variation of the shadowing $\sigma_{i}$ is dependent on transmission frequency and power level of landmark $i$. We will discuss its impact on localization performance in the next section.

The path loss propagation model indicates that the received signal strength is affected by the transmission frequency and power level. We focus on characterizing the impact of transmission frequency and power level on RSSbased localization. We next derive the Cramér-Rao Lower Bound (CRLB) of the localization error by taking into consideration of the transmission frequency and power level.

\subsection{CRLB of RSS-Based Localization}

CRLB sets a lower limit for the variance (or covariance matrix) of any unbiased estimates of an unknown parameter, which is widely used to analyze the localization performance [14], [9], [15]. We next derive the CRLB of RSSbased localization based on the frequency dependent path loss propagation model.

The CRLB under different transmission frequencies and power levels can be derived:

$$
\operatorname{Var}(\hat{\theta})=\frac{\sum_{i=1}^{N} \frac{\rho_{i}}{d_{i}^{2}}}{\sum_{i=1}^{N} \sum_{j=1, j \neq i}^{N} \rho_{i} \rho_{j} C_{i j}},
$$

where $C_{i j}=\left(\frac{\cos \varphi_{i} \sin \varphi_{j}}{d_{i} d_{j}}-\frac{\cos \varphi_{j} \sin \varphi_{i}}{d_{j} d_{i}}\right)^{2}$, and $\rho_{i}=\left(\frac{10 \beta_{i}}{\sigma_{i} \log 10}\right)^{2}$. The detailed derivation of Equation (2) is presented in Section 2 of the supplementary file available online.

According to (2), for a particular location, CRLB is affected by the parameter $\rho_{i}$, which is composed of shadowing variation $\sigma_{i}$ and path loss exponent $\beta_{i}$ from each landmark $i=1 \ldots N$. As shown in Section 2.1, $\rho_{i}=\left(\frac{10 \beta_{i}}{\sigma_{i} \log 10}\right)^{2}$ is dependent on the transmission frequency and power level of the wireless device. In order to achieve a lower CRLB, selecting appropriate frequency and power level to form a high-quality signal strength fingerprint for localization is critical. Thus, to achieve higher localization accuracy, we need to develop a selection strategy on transmission frequency and power level to generate highquality RSS fingerprints.

We define the impact factor as $\boldsymbol{\rho}=\left(\frac{10 \beta}{\sigma \log 10}\right)^{2}$, where $\boldsymbol{\rho}=\left[\rho_{1} \ldots \rho_{N}\right]$ is a $1 \times N$ vector and $N$ is the number of landmarks. The element of the impact factor $\rho_{i}$ can be considered as, $\rho_{i}=\not\left\{\rho_{i}^{1} \ldots \rho_{i}^{L_{p}}\right\}$, where $\left\{1 \ldots L_{p}\right\}$ are possible transmission frequencies and power levels measured at the $i$ th landmark. We call $L_{p}$ the dimension of landmark $i$. $\not L\{\cdot\}$ is a possible selection strategy, and $\rho_{i}$ is the selected set of frequencies and power levels for landmark $i$ after applying the selection strategy $\not L\{\cdot\}$. For example, at landmark 1, we have $L_{p}=6$ dimensions representing 6 different transmission frequencies and power levels including the combinations between two frequencies $(900 \mathrm{MHz}, 400 \mathrm{MHz})$ and three power levels $(0 \mathrm{dBm}, 5 \mathrm{dBm}$ and $10 \mathrm{dBm}) . \rho_{1}=\not L\left\{\rho_{1}^{1} \ldots \rho_{1}^{6}\right\}$, which is selected from the 6 dimensions based on the selection strategy $\not\{\cdot\}$. After applying the selection strategy, the resulting $\rho_{1}$ is a subset of the original possible transmission frequencies and power levels, e.g., $\rho_{1}=\{900 \mathrm{MHz}$, $5 \mathrm{dBm}\}$. More discussions are presented in Section 2 of the supplementary file available online.

The analysis of CRLB shows the feasibility of improving localization accuracy by selecting transmission frequency and power level to generate high-quality signal fingerprint for RSS-based localization. The localization accuracy is bounded by key parameters including distance to landmarks, path loss exponent, and variance of shadowing. In order to capture the impact of these parameters on localization accuracy, we focus on designing selection metrics and strategies that can quantify the impact of these parameters on localization performance.

\section{Selection Metrics on Localization ALGORITHMS}

Based on our theoretical analysis, we design selection metrics to improve the localization performance under different frequencies and power levels. We develop two selection metrics deviation and residual, which are used to select higher-quality RSS fingerprints. We then describe a broad class of localization algorithms that can benefit from the selection metrics. It ranges from lateration based, to fingerprint matching, to probabilistic based, and to Bayesian Networks. We further provide a theoretical analysis on how the linear least squares method benefits from the proposed selection metrics in Section 3 of the supplementary file available online.

\subsection{Terminology}

We use the following definitions and terms to introduce the localization algorithms and selection metrics. The fingerprints (i.e., RSS readings) are generated at multiple locations. For a targeting device at location $j$, the fingerprint is represented as a vector $\left(\left(x_{j}, y_{j}\right), S_{j}\right)$ with $\left(x_{j}, y_{j}\right)$ as the location $j$ where the RSS vector is collected. For 
example, $S_{j}=\left(s_{1 j}^{L_{1}}, \ldots, s_{1 j}^{L_{q}}, \ldots, s_{i j}^{L_{1}}, \ldots, s_{i j}^{L_{q}} \ldots, s_{n j}^{L_{1}}, \ldots, s_{n j}^{L_{q}}\right)$ represent the RSS vector from $n$ landmarks with $L_{q}$ dimensions: element $s_{i j}^{L_{p}}$ represents the RSS reading of a targeting device at location $j$ from the $i$ th $(i=1 \ldots n)$ landmark on the $L_{p}$ th $\left(L_{p}=L_{1} \ldots L_{q}\right)$ dimension. In this work, a dimension represents each frequency and power level combination at a landmark. We note that certain localization algorithms need training data to build signal maps during the offline phase, which is used for the online real-time localization. We use the fingerprints collected from multiple locations to form the training data and denote $T$ as the training set with multiple vectors $\left(\left(x_{j}, y_{j}\right), S_{j}\right)$ for $j=1 \ldots m$, and $m$ is the number of locations used for training.

\subsection{Selection Metrics}

Choosing the best fingerprint among the many possible combinations requires a characterization of the input RSS values. In this section we describe the two metrics we use for selection; the deviation of $R S S$ readings and residual. A given combination of RSS values can be evaluated according to these metrics, and the best one is selected as input to the localization algorithm.

\section{Deviation of RSS Readings}

We define the deviation of RSS readings as the measurement of the Root Mean Square Error (RMSE) when fitting the RSS readings to the signal propagation model for each individual dimension under one landmark. Thus, for a targeting device at location $j$ in the $L_{p}$ th dimension of landmark $i$, the deviation of RSS readings can be represented as the RMSE of the estimated RSS $\hat{s}_{i j} L_{p}$ from the fitted signal propagation model with respect to the observed RSS $s_{i j}^{L_{p}}$ :

$$
\operatorname{RMSE}\left(\hat{S}_{i}^{L_{p}}\right)=\sqrt{\frac{1}{m} \sum_{j=1}^{m}\left(\hat{s}_{i j}^{L_{p}}-s_{i j}^{L_{p}}\right)^{2}},
$$

where there are $m$ fingerprints, and $\hat{s}_{i j}^{L_{p}}$ is estimated by the signal to distance propagation model based on our prior work [17], and $s_{i j}^{L_{p}}$ is the observed RSS of the $L_{p}$ th dimension in landmark $i$ for $j=1 \ldots m$ locations.

\section{Residual}

The residual is represented as an error during the intermediate step of the localization process, which affects the final localization accuracy. Due to different localization principles, various algorithms have different residuals that are the leading factors contributing to the localization error. We next present our definition of residual for each algorithm together with the algorithm description in the following.

\subsection{Localization Algorithms}

\subsubsection{Lateration Based}

Localization using the lateration based approach is popular [8], [12], [13] and involves 2 steps: ranging and lateration.

\subsubsection{Ranging Step}

The purpose of the ranging step is to estimate the distance $d_{i}$ from the position of the targeting device $\theta=(x, y)$ to the $i$ th landmark $L_{i}=\left(x_{i}, y_{i}\right)$, which is represented as $d_{i}=\sqrt{\left(x_{i}-x\right)^{2}+\left(y_{i}-y\right)^{2}}$. A variety of physical modalities can be used to perform the ranging step such as Received Signal Strength (RSS), Time Of Arrival (TOA), Time Difference Of Arrival (TDOA) and hop count. In this work, we employ RSS to perform ranging. In particular, we use the linear regression approach in our prior work [17] to obtain the estimated distance $\hat{d}_{i}$.

\section{Lateration Step}

There are two popular methods, Nonlinear Least Squares (NLS) and Linear Least Squares (LLS), to get the estimated position of the target device $\hat{\theta}=(\hat{x}, \hat{y})$. In NLS, from the estimated distance $\hat{d}_{i}$ and known positions $L_{i}=\left(x_{i}, y_{i}\right)$ of the landmarks, the position $(x, y)$ of the target device can be estimated by finding $(\hat{x}, \hat{y})$ satisfying:

$$
(\hat{x}, \hat{y})=\operatorname{argmin}_{x, y} \sum_{i=1}^{n}\left[\sqrt{\left(x_{i}-x\right)^{2}+\left(y_{i}-y\right)^{2}}-\hat{d}_{i}\right]^{2},
$$

where $i=1 \ldots n$ for $n$ total number of landmarks. NLS usually requires high computational complexity and is difficult to analyze.

NLS can be approximated by solving a set of linear equations [6] as $A \hat{\theta}=b$, where:

$$
A=\left(\begin{array}{cc}
x_{1}-\frac{1}{n} \sum_{i=1}^{n} x_{i} & x_{n}-\frac{1}{n} \sum_{i=1}^{n} x_{i} \\
\vdots & \vdots \\
y_{1}-\frac{1}{n} \sum_{i=1}^{n} y_{i} & y_{n}-\frac{1}{n} \sum_{i=1}^{n} y_{i}
\end{array}\right)
$$

and

$$
b=\frac{1}{2}\left(\begin{array}{c}
\left(x_{1}^{2}-\frac{1}{n} \sum_{i=1}^{n} x_{i}^{2}\right)+\left(y_{1}^{2}-\frac{1}{n} \sum_{i=1}^{n} y_{i}^{2}\right) \\
-\left(\hat{d}_{1}^{2}-\frac{1}{n} \sum_{i=1}^{n} \hat{d}_{i}^{2}\right) \\
\vdots \\
\left(x_{n}^{2}-\frac{1}{n} \sum_{i=1}^{n} x_{i}^{2}\right)+\left(y_{n}^{2}-\frac{1}{n} \sum_{i=1}^{n} y_{i}^{2}\right) \\
-\left(\hat{d}_{n}^{2}-\frac{1}{n} \sum_{i=1}^{n} \hat{d}_{i}^{2}\right)
\end{array}\right) .
$$

$A$ is described by the coordinates of landmarks, and $b$ is composed of estimated distances to the landmarks and the coordinates of landmarks. We call the above formulation of the problem Linear Least Squares(LLS). The position estimation of the targeting device is done by solving $\hat{\theta}=\left(A^{T} A\right)^{-1} A^{T} b$.

\section{Residual}

We define the residual $R$ of the lateration method as the averaged difference between the estimated distance to each landmark $\hat{d}_{i}$ and the distance of the localized position to each landmark $\left\|\hat{\theta}-L_{i}\right\|$. The residual is represented as

$$
R=\frac{1}{n} \sum_{i=1}^{n}\left(\hat{d}_{i}-\left\|\hat{\theta}-L_{i}\right\|\right)^{2}
$$

Intuitively, we expect the smaller residual can lead to better localization accuracy. The analysis of the relationship between the residual and the localization error is presented in Section 3 of the supplementary file available online.

\subsubsection{Fingerprint Matching}

The Radar algorithm [2] is a classic machine learning method based on fingerprint matching, which requires 


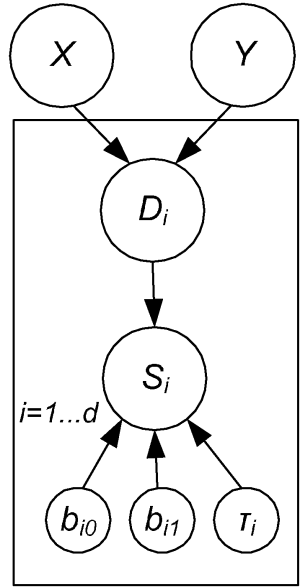

Fig. 1. Bayesian graphical model in our study.

building a signal map consisting of RSS fingerprints with known $(x, y)$ locations. On top of the basic fingerprints in RADAR, Gridded Radar (GR) uses the IMG (Interpolated Map Grid) to build an interpolated signal map. GR builds a regular grid of tiles over the localization area that describes the expected fingerprint for the area described by each tile. Given a RSS fingerprint of a targeting device, GR returns the position $(x, y)$ of the closest fingerprint in the interpolated signal map to the one of the targeting device as the location estimation, where closest means the Euclidean distance of the fingerprints in the signal space.

\section{Residual}

Given the observed fingerprint of a targeting device at location $j$, the residual of Gridded Radar is defined as the Euclidean distance of the fingerprint from the targeting device to the interpolated signal map in signal space:

$$
R=\sqrt{\sum_{i=1}^{n} \sum_{\text {col }=1}^{\text {col } M} \sum_{\text {row }=1}^{\text {row } M} \| s_{i j}^{L_{p}}-S G_{i}^{L_{p}}(\text { row }, \text { col }) \|^{2}}
$$

where $n$ is the number of landmarks, $s_{i j}^{L_{p}}$ is the RSS reading of the targeting device at location $j$ in the $L_{p}$ th dimension of the $i$ th landmark where $L_{p}=L_{1} \ldots L_{q}$ with $q$ as the total number of dimensions under that landmark, and $S G_{i}^{L_{p}}$ represents the interpolated RSS matrix of the $L_{p}$ th dimension for the $i$ th landmark, where row is the total number of rows in the interpolated RSS matrix with row $=1 \ldots$ row $M$ and $\mathrm{col}$ is the total number of columns in the interpolated RSS matrix with $\operatorname{col}=1 \ldots \operatorname{col} M$. We note that for different landmark $i$, dimension $L_{p}$ can be different.

\subsubsection{Probabilistic Based}

In this work, we choose to study Area Based Probability (ABP) [8], which is a representative method based on the statistical Bayes' Rule to perform location estimation. ABP also uses the interpolated signal map as in GR and computes the likelihood of the observed fingerprint of a targeting device matching a fingerprint of each tile in the interpolated signal map. It returns the top probability tile set whose sum matches the desired confidence level $\alpha$. In particular, by using Bayes' rule, ABP computes the probability of being at each tile $T_{j}$ on the floor given the fingerprint of the targeting device $S_{T}$ :

$$
P\left(T_{j} \mid S_{T}\right)=\frac{P\left(S_{T} \mid T_{j}\right) \times P\left(T_{j}\right)}{P\left(S_{T}\right)}
$$

\section{Residual}

Since the number of tiles in the returned tile set represents how well the algorithm captures the true location of the targeting device, the less the number of returned tiles indicates the better the probabilistic matching is found. We thus define the residual of $\mathrm{ABP}$ as the number of tiles returned based on the confidence level $\alpha$.

\subsubsection{Bayesian Networks}

Bayesian Networks [8] utilizes the Bayesian Graphical Model to compute the distribution of the position $(x, y)$ of a targeting device. In particular, Bayesian Networks encodes the relationship between the RSS readings and the location based on the signal-distance propagation model. The initial parameters of the model are unknown, and the training set collected from multiple known locations is used to adjust the parameters of the model according to the relationships encoded in the network.

Fig. 1 depicts the basic Bayesian Graphical Model The random variables $s_{i}, i=1 \ldots n$ denotes the expected signal strength of the corresponding dimension (e.g., a combination of frequency and power level) in the landmark $i$. The values of these random variables depend on the Euclidean distance $d_{i}$ between the landmark's location $\left(x_{i}, y_{i}\right)$, and the location where the signal $s_{i}$ is measured $(x, y)$. The baseline expected value of $s_{i}$ follows a signal propagation model $s_{i}=b_{0 i}+b_{1 i} \log \left(d_{i}\right)$, where $b_{0 i}, b_{1 i}$ are the parameters specific to each $i$. The distance $d_{i}=\sqrt{\left(x-x_{i}\right)^{2}+\left(y-y_{i}\right)^{2}}$ in return depends on the location $(x, y)$ of the measured signal. The network models noise and outliers by modeling the expected value, $s_{i}$, as a $t$-distribution around the above propagation model, with variance $\tau_{i}, s_{i} \sim t\left(b_{0 i}+b_{1 i} \log d_{i}, \tau_{i}, 2\right)$. Using the training fingerprints $T$, the network can learn the specific values for all the unknown parameters $b_{0 i}, b_{1 i}, \tau_{i}$ and the joint distribution of $(x, y)$ location of the targeting device.

In general, there is no closed form solution for the returned joint distribution of the $(x, y)$ location. Therefore, we use a Markov Chain Monte Carlo (MCMC) simulation approach to draw samples from the joint density, and then pick the samples that give a 95 percent confidence on the density. Finally, we approximate the returned area by the tiles where those samples fall.

\section{Residual}

When computing the joint distribution of the location of the targeting device, we define the residual as: $R=\sqrt{\sigma_{x}^{2}+\sigma_{y}^{2}}$, where $\sigma_{x}$ and $\sigma_{y}$ are the standard deviation of the position variable $x$ and $y$, which represents how stable the estimated position distribution is. Intuitively, the more stable the distribution of the estimated position is, we expect better localization accuracy. 


\section{Selection Strategy and Simulation RESULTS}

In this section, we first present our dimension selection strategies and selection procedures to characterize the impact of multiple frequencies and multiple power levels on performing localization. To validate our proposed approaches, we then simulate a wireless indoor environment, and compare the theoretical CRLB with simulation results.

\subsection{Selection Strategies}

We next develop three selection methods, namely Whole Dimension, Matching Characteristic, and Constrained Landmark, which utilize different combination of multiple frequencies and multiple power levels to perform localization.

\section{Whole Dimension}

This is a straightforward selection method. Each combination of a frequency and a power level from an individual landmark is treated as one dimension. Given there are 5 landmarks and 6 possible combinations from each landmark in our experimental setup, there are total 30 dimensions. In this selection method, all the dimensions are used to construct a fingerprint. For instance, $S=\left[s_{1}^{1}, \ldots, s_{1}^{6}, \ldots, s_{i}^{1}, \ldots, s_{i}^{6}, \ldots s_{5}^{1}\right.$, $\left.\ldots, s_{5}^{6}\right]$ is the fingerprint for a testing point when the Whole Dimension selection method is used. This method may incur high computational cost for certain algorithms such as BN since it needs to compute the joint position distribution across high dimensions.

\section{Matching Characteristic}

In our experiments, each dimension has a fixed frequency and power level, which we call a signal characteristic. In contrary to the Whole Dimension method, the Matching Characteristic method tries to keep the number of dimensions as the same as the number of landmarks when selecting RSS fingerprints. In the Matching Characteristic selection method, we request the same characteristic to be chosen from the 6 dimensions for each landmark, that is, during one selection process for localization, the combination of the frequency and power level selected should be the same across all landmarks. Based on this selection metric, there are total 6 possible combinations of frequency and power level. To perform localization, Matching Characteristic will use our selection metrics to determine what is the appropriate combination of the frequency and power level to be chosen as the characteristic for each testing point in our experiments.

When Matching Characteristic selection is applied using RMSE, RMSE will be calculated for each combination of the frequency and power level and compare across all the 6 possible combinations. Within each combination, we can first calculate $R M S E_{i}^{L_{p}}(\hat{S})$ for dimension $L_{p}$ of landmark $i$ based on Equation (3). We then calculate the RMSE for this combination over all the landmarks as $R M S E_{L_{p}}(\hat{S})=\sqrt{\sum_{i=1}^{n} R M S E_{i}^{L_{p}(\hat{S})^{2}}}$ with $L_{p}=1 \ldots 6$. The RSS fingerprints from the frequency and power level combination that produces the minimum value of RMSE
$R M S E_{\min }=\min _{L_{p}}\left(R M S E_{L_{p}}(\hat{S})\right)$ over all the combinations will be selected as the appropriate characteristic for performing localization.

On the other hand, When Matching Characteristic selection is applied using residual, the residual is calculated for all the frequency and power level combinations. We choose the frequency and power level combination that produces the minimum residual as characteristic to perform localization for each algorithm. For different testing points, the selected characteristic may be different, because it is possible that different testing points have different combinations of frequency and power level that produces the minimum residual.

\section{Constrained Landmark}

Instead of using the full dimension of the data, the objective of the Constrained Landmark takes advantage of using multiple frequencies and multiple power levels, but without increasing the dimension for algorithm computation and without fixing the characteristics across landmarks. 'Constrained' means that the RSS fingerprint must be constructed by selecting from different landmarks. However, the dimension chosen in each landmark could be different from different landmarks. Particularly, the constructed RSS fingerprint is composed of element $S_{i}^{L_{p}}$ with $i=1 \ldots 5$ in our experiments, and for each landmark $i$, the dimension $L_{p}$ can be selected differently corresponding to different combinations of frequency and power level. Therefore, there can be total $6^{5}=7776$ different combinations when applying Constrained Landmark selection in our experiments. The computational cost of using Constrained Landmark is higher than using Matching Characteristic.

This selection method will be used together with our selection metrics to perform localization by each algorithm. When the Constrained Landmark selection method is applied by using RMSE, the same calculation of RMSE will be performed as described in the Matching Characteristic selection to search for the frequency and power level combination with the minimum RMSE, however, there are total $6^{5}$ combinations. Likewise, when the Constrained Landmark selection method is applied by using residual, the same computation of residual will be performed as presented in Matching Characteristic. The combination of the frequency and power level that produces the minimum residual across the $6^{5}$ combinations will be selected.

\subsection{Simulation Methodology}

We conduct simulations to validate our proposed approaches by comparing the theoretical CRLB with simulation results. In the simulation, the RSS measurements are collected from the wireless device placed at 100 uniformly distributed locations in a 100 feet by 100 feet area. The landmarks are deployed at four corners of the area of interest. In order to simulate the indoor signal propagation, the path loss propagation model discussed in Section 2 is utilized. We perform localization on the wireless device for 200 times when the device is placed at each position. We then calculate the CRLB and the average Root Mean Square (RMS) error of location estimates. Two selection metrics (in 
TABLE 1

Parameter Setup for Simulation

\begin{tabular}{|c|c|c|c|c|c|c|}
\hline \hline & Dim 1 & Dim 2 & Dim 3 & Dim 4 & Dim 5 & Dim 6 \\
\hline$\beta$ & 3.2 & 2.74 & 2.52 & 2.17 & 1.84 & 1.39 \\
\hline$\sigma$ & 1.4 & 1.6 & 2 & 2.52 & 3 & 3.64 \\
\hline$\beta / \sigma$ & 2.28 & 1.71 & 1.26 & 0.86 & 0.61 & 0.38 \\
\hline$P\left(d_{0}\right)$ & 10 & 10 & 5 & 5 & 0 & 0 \\
\hline
\end{tabular}

Section 3.2) and three selection strategies (in Section 4.1) are applied to each localization attempt.

\section{Dimensions}

Each dimension is a unique combination of one frequency and one power level. Different dimensions have different $\frac{\beta}{\sigma}$ (the impact factor $\left.\rho=\left(\frac{10 \beta}{\sigma \log 10}\right)^{2} \propto\left(\frac{\beta}{\sigma}\right)\right)$ or $P\left(d_{0}\right)(\mathrm{dBm})$ values [1], [7] as discussed in Section 2. The parameters of $\frac{\beta}{\sigma}$ and $P\left(d_{0}\right)$ used in simulation are summarized in Table 1 . To study how the number of dimensions involved in the selection strategy affect the localization performance, three different scenarios are tested: 1) Dim 1 6 are involved in selection; 2) Dim 1 4 are involved in selection; and 3) only Dim 1 and Dim 2 are involved in selection. For each scenario, the Whole Dimension means that all dimensions are used to perform localization, i.e., six, four, and two dimensions are used in these three scenarios respectively.

\subsection{Simulation Results}

\section{Metrics}

The localization performance of the proposed mechanisms is evaluated by average location RMS error, which is the averaged root mean square (RMS) of location error for all 100 locations over 200 localization attempts at each location.

\section{Localization Accuracy Under Different Number of Dimensions}

We evaluate the average location RMS error over different number of dimensions with three selection strategies and two selection metrics, and compare it with the theoretical CRLB. Fig. 2 shows the CRLB bounds the localization error of the two algorithms, lateration based and fingerprint matching. CRLB is a lower bound for unbiased estimators. The lateration based algorithm (linear least square algorithm) is unbiased, while the fingerprint matching algorithm may be biased due to the limited number of training points that the locations are matched to [4]. Therefore, the localization error for lateration method is more approaching to CRLB. We observe that the derived CRLB bounds the performance of both localization algorithms for lateration based and fingerprint matching algorithms. Particularly, for lateration based method, as shown in Figs. $2 a$ and $2 b$, the theoretical CRLB is lower than the simulation results

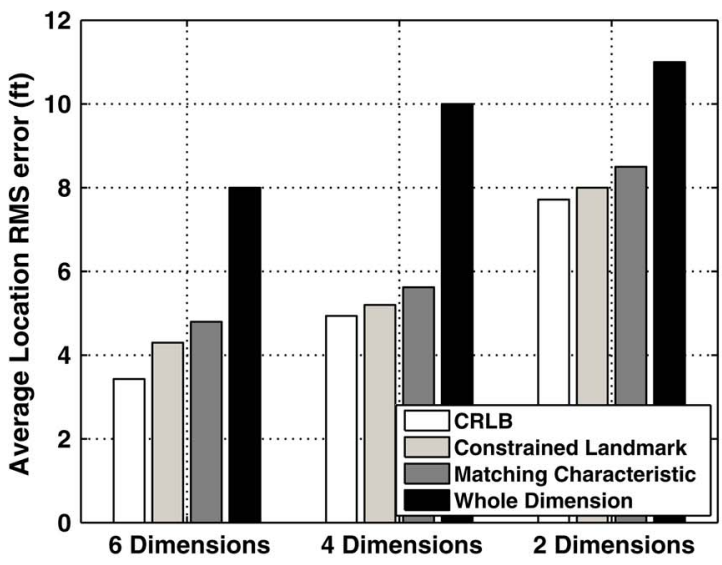

(a)

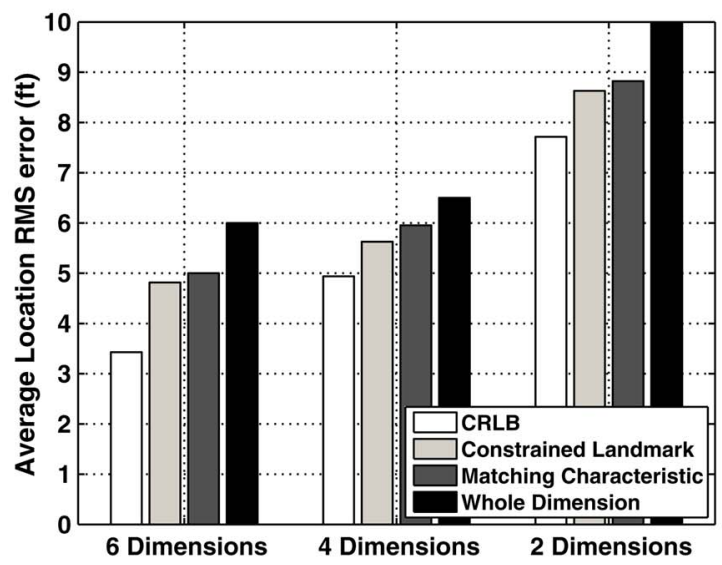

(c)

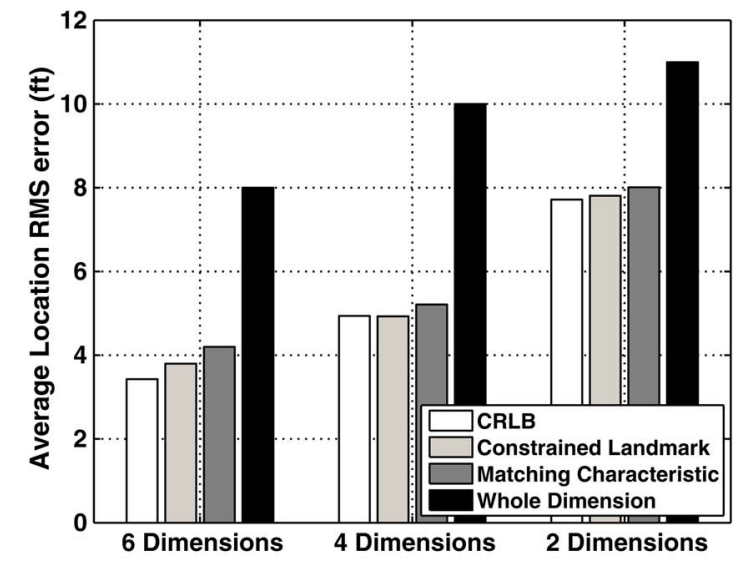

(b)

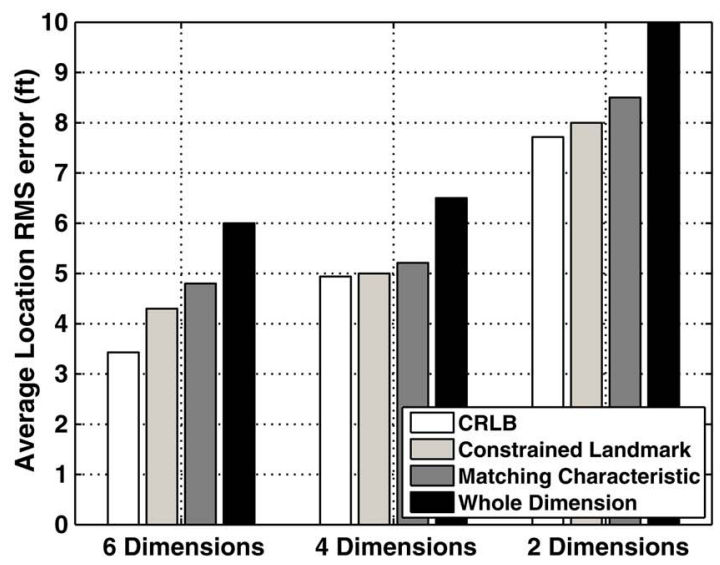

(d)

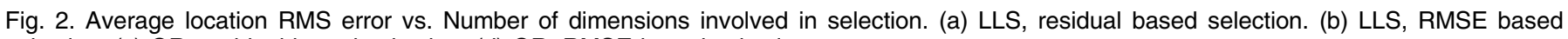
selection. (c) GR, residual based selection. (d) GR, RMSE based selection. 


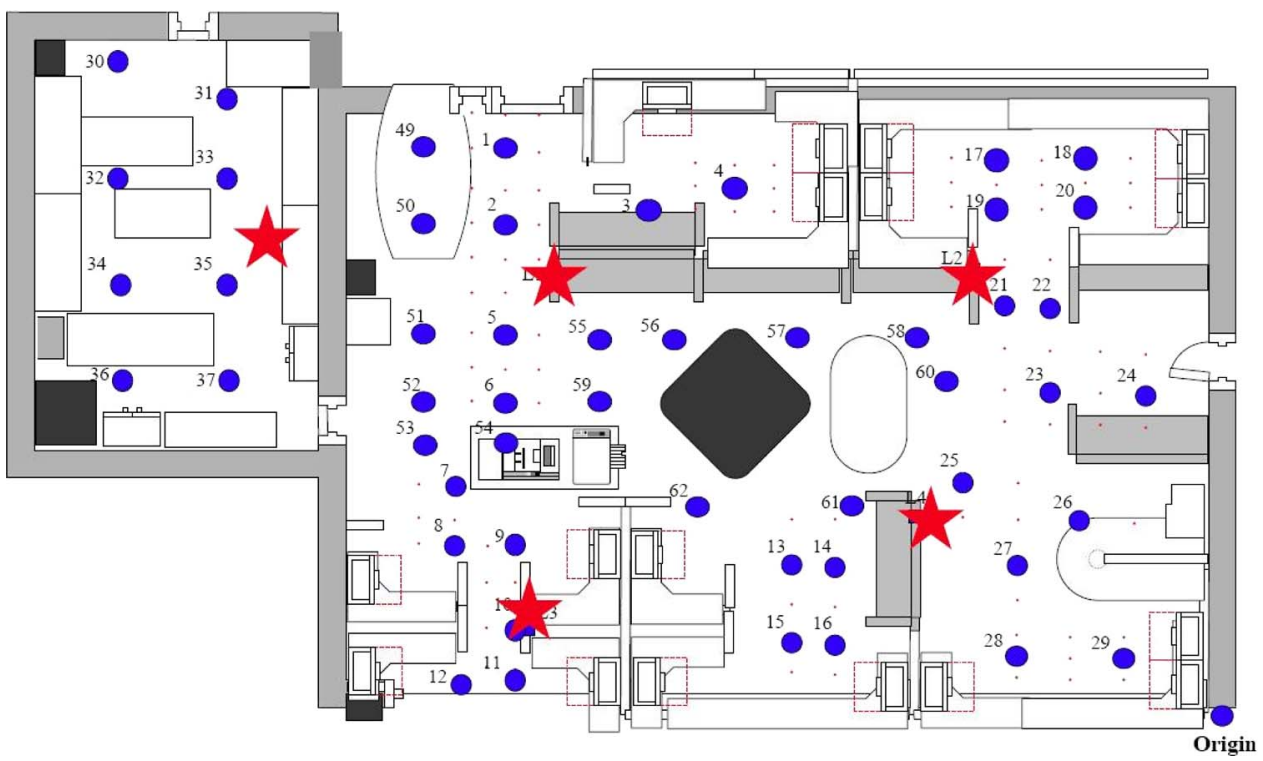

Fig. 3. Burchard 2nd floor map.

under all the scenarios for both selection metrics including residual and RMSE.

Furthermore, with more dimensions involved in the selection, lower average location RMS error can be achieved as shown in Fig. 2. This is because more dimensions bring higher possibilities for our selection based approach to choose higher quality fingerprint. Particularly, for lateration based method as shown in Figs. 2a and 2b, the average error improvement is around 50 percent (from $8 \mathrm{ft}$ to $4 \mathrm{ft}$ under 6 dimensions scenario); while for fingerprint matching method as shown in Figs. $2 \mathrm{c}$ and $2 \mathrm{~d}$, we observe around 20 percent improvement in averaged error (from $6 \mathrm{ft}$ to $5 \mathrm{ft}$ under 6 dimensions scenario). We find that the lateration based algorithm benefits more from our selection strategies than fingerprint matching algorithm. Further, the fingerprint matching algorithm is more robust than lateration based algorithm. In addition, we observe that the localization performance of our proposed selection based methods outperforms that of without selection substantially. For these two selection metrics, RMSE based selection metric slightly outperforms the residual based metric. For our selection strategies: Constrained Landmarks and Matching Characteristic, they show similar trend on improving localization performance. As described in Section 3, choosing between the selection strategies has a tradeoff between accuracy and computational cost.

\section{Experimental Methodology and Evaluation Results}

We next evaluate our approach in practice. In this section, we first describe our experimental methodology by presenting the testbed infrastructure and the data collection process. We then experimentally evaluate the impact of multiple frequencies and multiple power levels on the performance of localization algorithms.

\subsection{Testbed Infrastructure and Data Collection}

RSS measurements are conducted using a localization testbed [5] with active RFID tags and readers from InPoint
[18]. We collect experimental data on the second floor of Buchard building at Stevens Institute of Technology, which is a $70 \mathrm{ft} \times 80 \mathrm{ft}$ area as shown in Fig. 3. This is a large lab area containing office wall dividers and furniture, such as desks, shelves and chairs. There are many electronic devices and lab equipment in our experimental area, e.g., desktops, laptops, smart phones, and spectrum analyzers. We deploy 5 landmarks and collect RSS fingerprints for 51 testing points. Landmarks and testing positions are shown as stars and dots respectively. We program the RFID tag to transmit between two frequencies, $400 \mathrm{MHz}$ and $900 \mathrm{MHz}$, alternatively. Data collection is then repeated for 3 transmission power levels $(0 \mathrm{dBm}, 5 \mathrm{dBm}$, and $10 \mathrm{dBm})$ at 2 frequencies ( $400 \mathrm{MHz}$ and $900 \mathrm{MHz}$ ) by placing three transmitters at the same testing location, which approximates a system with 6 different dimensions (power level and frequency combinations). Given the number of testing points and the dimension of each testing point, the total number of fingerprints in our experimental dataset is $51 \times 30=1530$. More details of our experimental methodology are presented in Section 4 of the supplementary file available online.

\subsection{Performance Baseline}

Based on our data collection using multiple frequencies (i.e., $400 \mathrm{MHz}$ and $900 \mathrm{MHz}$ ) and multiple power levels (i.e., $0 \mathrm{dBm}, 5 \mathrm{dBm}$, and $10 \mathrm{dBm}$ ), we have total 6 single dimension RSS readings for each landmark. We can perform localization by using each individual dimension, which has a fixed frequency and a fixed power level (e.g., $900 \mathrm{MHz}$ and $0 \mathrm{dBm}$ ). We use the localization performance from running these 6 single dimension data sets to report baseline results, which will be used to compare with those from different combination of frequency and power level through selection.

\subsection{Results}

We study all the algorithms described in Section 3.3 and evaluation is conducted using both selection metrics (RMSE and Residual) and all three selection strategies (Whole Dimension, Constrained Landmark, and Matching Characteristic). 


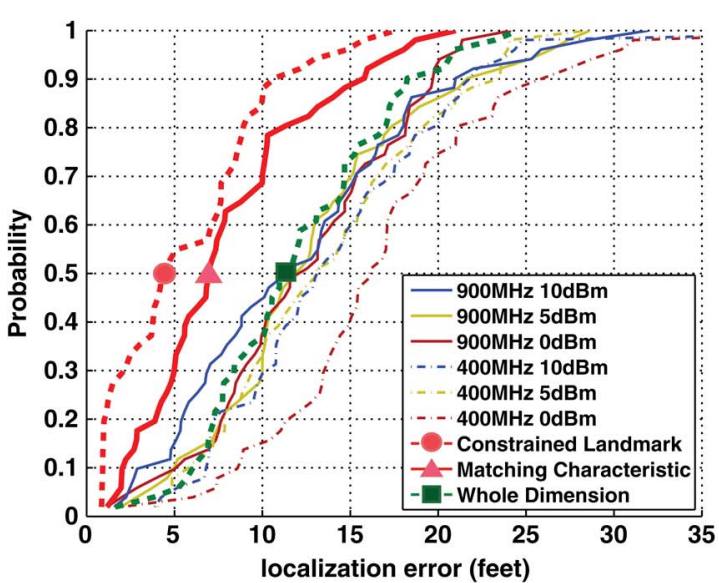

(a)

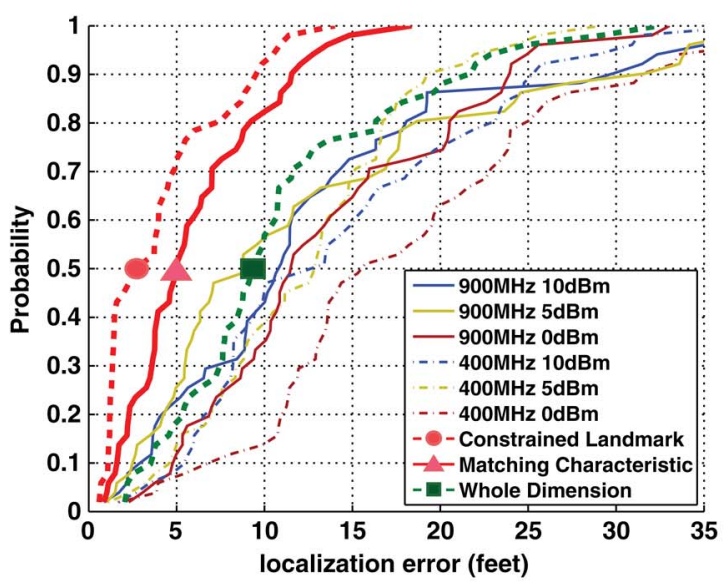

(c)

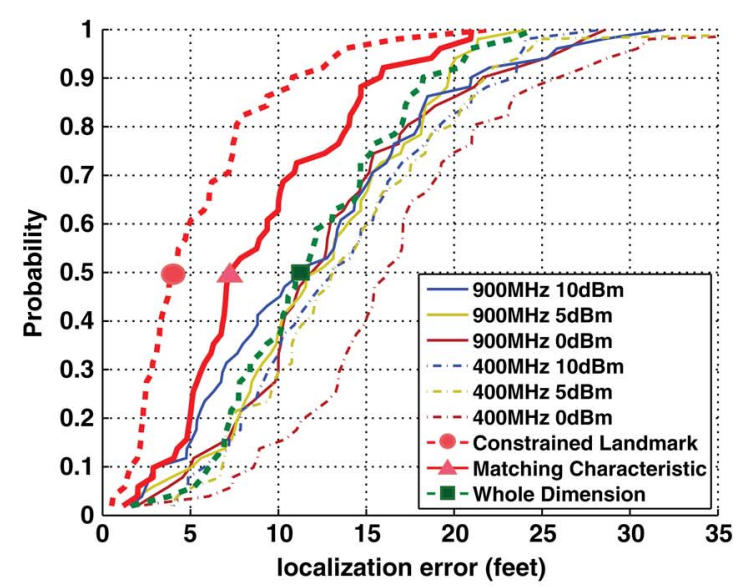

(b)

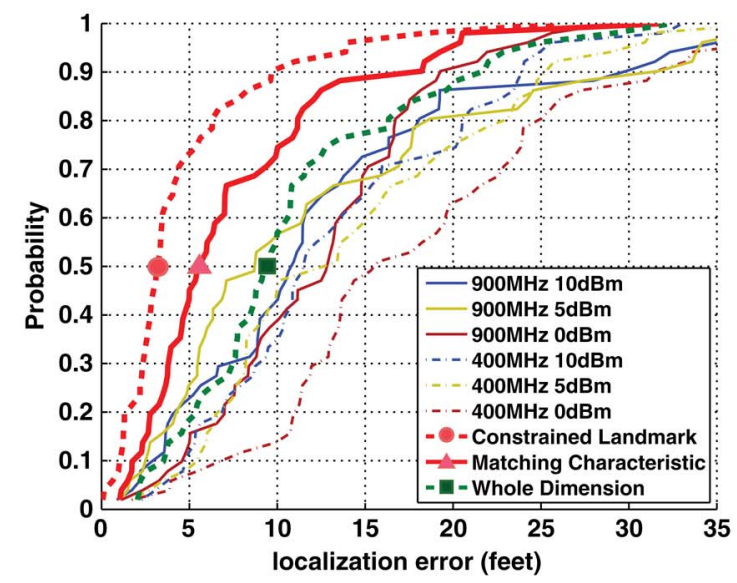

(d)

Fig. 4. Lateration based algorithms: Localization accuracy CDF under different selection strategies. (a) LLS, Residual based selection. (b) LLS, RMSE based selection. (c) NLS, Residual based selection. (d) NLS, RMSE based selection.

In all our studies, localization performance is characterized by the Accuracy metric, which is the Euclidean distance between the estimated location from the algorithm and the device's actual location.

\section{Lateration Based Algorithms}

Fig. 4 presents the localization accuracy Cumulative Distributed Function (CDF) for lateration based algorithms under different selection strategies. Results are included for both LLS and NLS algorithms, and for both residual and RMSE based selection metrics. Performance for each single dimension is also plotted as our baseline for comparison.

Firstly, we notice that the accuracy CDFs from all 6 single dimensions are similar to each other. This indicates that simply changing the dimension characteristics, for example from using one transmitting frequency to another, is unlikely to offer much accuracy improvements. Secondly, contrary to our initial expectations, although using Whole Dimension achieves a performance close to the best among all the single dimensions, the additional information does not bring significant accuracy improvements for lateration based algorithms. This actually is directly related to how this set of algorithms is designed. For lateration based algorithms, path loss signal propagation model is enforced for distance estimation with a low accommodation of the noise from the collected data. Noisy RSS information thus directly translates to uncertainty in the lateration phase. By increasing the number of dimensions in this selection approach, the distance estimation adds more uncertainties during the lateration phase, however no further geometrical constraints are added to help reduce the error. Thus, simple least squares metric is not sufficient to cope with the additional uncertainty.

Finally, compliant to our CRLB analysis and simulation results, the intelligent selection strategies based on both metrics successfully achieve better localization accuracy for lateration based algorithms. For example, using Constrained Landmark with residual metric, LLS's median error decreases from $12 \mathrm{ft}$ to $5 \mathrm{ft}$ (58 percent improvement) and the 90th percentile error changes from $22 \mathrm{ft}$ to $10 \mathrm{ft}$ (54 percent improvement). Similarly, using Matching Characteristics with RMSE metric, LLS's median error decreases from $12 \mathrm{ft}$ to $7 \mathrm{ft}$ (42 percent improvement) and the 90th percentile error changes from $22 \mathrm{ft}$ to $15 \mathrm{ft}$ (32 percent improvement). We also notice that Constrained Landmark significantly outperforms Matching Characteristics and always performs the best among all the selection strategies. Such accuracy gain however, as pointed in Section 3, comes with significantly more computational cost.

In summary, for lateration based algorithms, it is not sufficient to simply deploy multi-frequency and multipower levels and expect performance improvements. 


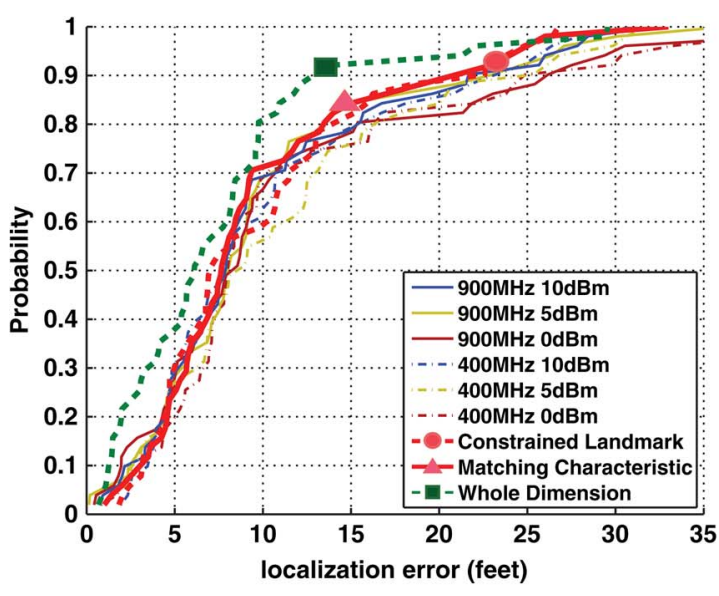

(a)

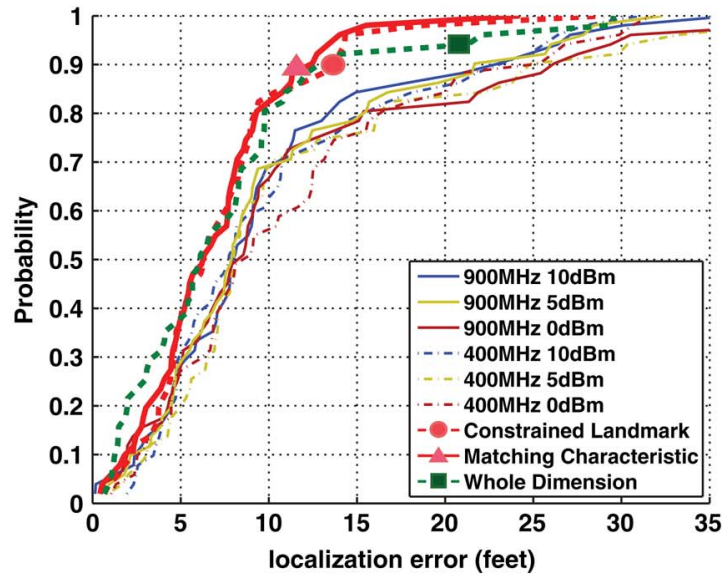

(b)

Fig. 5. Fingerprint Matching algorithm (GR): Localization accuracy CDF under different selection strategies. (a) Residual based selection. (b) RMSE based selection.

Intelligent selection strategies are required to prune the RSS measurements to better utilize the additional dimensions.

\section{Fingerprint Matching Algorithm (GR)}

Fig. 5 shows the localization accuracy CDFs of Gridded Radar algorithm while using residual and RMSE as metric under different selection strategies. We again observe that performance for single dimension consistently agrees with each other, indicating no improvement by simply changing transmission power or frequency. Whole Dimension, however, outperforms single dimensions. Specifically, the median error decreases from $8 \mathrm{ft}$ to $6 \mathrm{ft}$ (20 percent improvement) and the 90th percentile error shrinks from $25 \mathrm{ft}$ to $13 \mathrm{ft}$ ( 48 percent improvement). This is because GR is a machine learning based matching algorithm which straightforwardly benefits from a more precise signal map generated by adding more dimensions.

Furthermore, the intelligent selection strategies, Constrained Landmark and Matching Characteristic, tend to improve the performance over single dimensions. Although they do not necessarily offer much more accuracy advantage over using full dimension directly, the lower computational cost, especially for Matching Characteristic, compared to Whole Dimension makes them attractive for achieving better localization than using single dimensions only. We also notice that RMSE based selection strategies perform better than residual based selection strategies. Overall, for GR algorithm, deploying multi-frequency and multi-power can bring around significant performance improvements. There is also a tradeoff between localization accuracy and computational cost when deciding on different selection strategies. The results of probabilistic based algorithm (ABP) and Bayesian Networks (BN), together with a study on the distribution of selection metrics and strategies are presented in Section 4 of the supplementary file available online.

\section{Conclusion}

In this paper, we explore improving wireless localization performance in face of multipath effects by utilizing multiple frequencies and transmission powers. By studying the Cramér-Rao lower Bound of localization using RSS measurements, we investigate the feasibility of applying selection from multiple frequencies and power levels to improve localization accuracy. Based on the theoretical analysis through CRLB, we study a broad range of algorithms employing received signal strength (RSS) to perform localization including lateration based, fingerprint matching, probabilistic based, and Bayesian Networks. We develop three selection strategies, Whole Dimension, Matching Characteristic, and Constrained Landmark. Our selection strategies work with selection metrics, e.g., deviation of RSS readings and residual, to form high quality RSS fingerprints out of multiple dimensions resulting from the use of multifrequency and multi-power.

We perform both theoretical analysis as well as validation of our approach on localization algorithms using simulation and real experiments. Our analysis on the Linear Least Squares (LLS) algorithm show that selection should improve the localization performance. Our simulation results in indoor environments confirm the effectiveness of our proposed approach. Furthermore, our experimental study on a localization testbed with RFID transmitters varying the frequency and power showed that using multiple frequencies and power levels improve the performance of all the algorithms under study. We also find that different algorithms react differently to the selection strategies. Lateration based algorithms are the most sensitive to selection, whereas algorithms rooted in machine learning are much more robust to adding dimensions. In addition, we observe there is a tradeoff between improving performance and reducing computational cost through intelligent selection.

\section{ACKNOWLEDGMENT}

This work is supported in part by the National Science Foundation Grants CCF-1018270, CNS-1016303, CNS-1318751, CNS-1318748, and ARO W911NF-13-1-0288. This work was done while H. Liu was at Stevens Institute of Technology.

\section{References}

[1] A. Affandi, G. Zein, and J. Citerne, "Investigation on Frequency Dependence of Indoor Radio Propagation Parameters," in Proc. VTC, 1999, pp. 1988-1992. 
[2] P. Bahl and V. Padmanabhan, "RADAR: An In-Building RFBased User Location and Tracking System," in Proc. IEEE INFOCOM, Mar. 2000, pp. 775-784.

[3] P. Barsocchi, S. Lenzi, S. Chessa, and Furfari, "Automatic Virtual Calibration of Range-Based Indoor Localization Systems," Wireless Commun. Mobile Comput., vol. 12, no. 17, pp. 1546-1557, Dec. 2012.

[4] G. Chandrasekaran, M. Ergin, J. Yang, S. Liu, Y. Chen, M. Gruteser, and R.P. Martin, "Empirical Evaluation of the Limits on Localization Using Signal Strength," in Proc. 6th Annu. IEEE Commun. Soc. Conf. SECON, June 2009, pp. 1-9.

[5] Y. Chen, G. Chandrasekaran, E. Elnahrawy, J. Francisco, K. Kleisouris, X. Li, R. Martin, R. Moore, and B. Turgut, "GRAIL: A General Purpose Localization System," Sens. Rev., Special Edition, Localisation Syst., vol. 28, no. 2, pp. 115-124, 2008.

[6] Y. Chen, J. Francisco, W. Trappe, and R.P. Martin, "A Practical Approach to Landmark Deployment for Indoor Localization," in Proc. 3rd Annu. IEEE Commun. Soc. Conf. SECON, Sept. 2006, pp. 365-373.

[7] B. Donlan, D. McKinstry, and R. Buehrer, "The UWB Indoor Channel: Large and Small Scale Modeling," IEEE Trans. Wireless Commun., vol. 5, no. 10, pp. 2863-2873, Oct. 2006.

[8] E. Elnahrawy, X. Li, and R.P. Martin, "The Limits of Localization Using Signal Strength: A Comparative Study," in Proc. 1st IEEE Int'l Conf. SECON, Oct. 2004, pp. 406-414.

[9] A. Hossain and W.-S. Soh, "Cramer-Rao Bound Analysis of Localization Using Signal Strength Difference as Location Fingerprint," in Proc. IEEE INFOCOM, Mar. 2010, pp. 1-9.

[10] G. Janssen, P. Stigter, and R. Prasad, "Wideband Indoor Channel Measurements and BER Analysis of Frequency Selective Multipath Channels at 2.4, 4.75, and $11.5 \mathrm{GHz},{ }^{\prime \prime}$ IEEE Trans. Commun., vol. 44, no. 10, pp. 1272-1288, Oct. 1996.

[11] Y. Kim and S. Li, "Capturing Important Statistics of a Fading/ Shadowing Channel for Network Performance Analysis," IEEE J. Sel. Areas Commun., vol. 17, no. 5, pp. 888-901, May 1999.

[12] K. Langendoen and N. Reijers, "Distributed Localization in Wireless Sensor Networks: A Quantitative Comparison," Comput. Netw., vol. 43, no. 4, pp. 499-518, Nov. 2003.

[13] D. Niculescu and B. Nath, "Ad Hoc Positioning System (APS)," in Proc. IEEE GLOBECOM, 2001, pp. 2926-2931.

[14] N. Patwari, J.N. Ash, S. Kyperountas, A.O. Hero, R.L. Moses, and N.S. Correal, "Locating the Nodes: Cooperative Localization in Wireless Sensor Networks," IEEE Signal Process. Mag., vol. 22, no. 4, pp. 54-69, July 2005.

[15] N. Patwari, A.O. Hero, M. Perkins, N.S. Correal, J. Robert, and R.J. O'Dea, "Relative Location Estimation in Wireless Sensor Networks," IEEE Trans. Signal Process., vol. 51, no. 8, pp. 21372148, Aug. 2003

[16] S. Seidel and T.S. Rappaport, "914 MHz Path Loss Prediction Models for Indoor Wireless Communications in Multifloored Buildings," IEEE Trans. Antennas Propag., vol. 40, no. 2, pp. 207-217, Feb. 1992.

[17] J. Yang and Y. Chen, "Indoor Localization Using Improved RSS-Based Lateration Methods," in Proc. IEEE Globecom, Nov. 2009, pp. 1-6.

[18] Y. Zhang, G. Bhanage, W. Trappe, Y. Zhang, and R. Howard, "Facilitating an Active Transmit-Only RFID System Through Receiver-Based Processing," in Proc. 4th Annu. IEEE Commun. Soc. Conf. SECON, 2007, pp. 421-430.

[19] X. Zheng, H. Liu, J. Yang, Y. Chen, J. Francisco, R.P. Martin, and X. Li, "Characterizing the Impact of Multi-Frequency and MultiPower on Localization Accuracy," in Proc. IEEE 7th Int'l Conf. MASS, Nov. 2010, pp. 156-165.

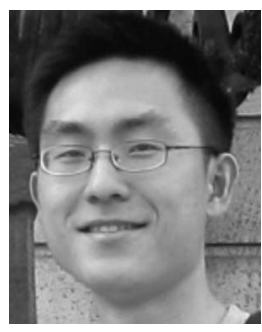

Xiuyuan Zheng received the bachelor's degree from the Department of Telecommunication Engineering at Nanjing University of Posts and Communications, China, in 2007. He is currently pursuing the $\mathrm{PhD}$ degree at the Electrical and Computer Engineering Department at Stevens Institute of Technology. His research interests include information security and privacy, wireless localization and location-based services (LBS), wireless and sensor networks. $\mathrm{He}$ is currently working in the Data Analysis and Information SecuritY (DAISY) Lab with Prof. Yingying Chen. He was in the Master program in the Electrical and Computer Engineering Department at Stevens Institute of Technology from 2007 to 2009.

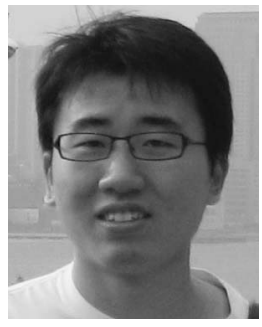

Hongbo Liu received the $\mathrm{PhD}$ degree in electrical engineering from Stevens Institute of Technology. Since August 2013, he was with IUPUI as an Assistant Professor in Department of Electrical and Computer Engineering. His research interests include mobile and pervasive computing, cyber security and privacy and smart grid. $\mathrm{He}$ is the recipient of the Best Paper Award from ACM MobiCom 2011 and the Outstanding Research Award in 2012 from Stevens Institute of Technology.

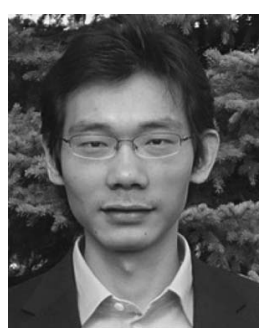

Jie Yang received the $\mathrm{PhD}$ degree in computer engineering from Stevens Institute of Technology in 2011. He is currently an Assistant Professor in the Department of Computer Science and Engineering at Oakland University. He was a postdoctoral fellow in the Data Analysis and Information Security (DAISY) Laboratory at Stevens Institute of Technology. His research interests include cyber security and privacy, mobile and pervasive computing, wireless localization systems, and mobile social networks. He is the recipient of the Best Paper Award from ACM International Conference on Mobile Computing and Networking (MobiCom) 2011 and the Outstanding Research Award in 2009 from Stevens Institute of Technology. His research has received wide press coverage including MIT Technology Review, The Wall Street Journal, CNET News, and Yahoo News.

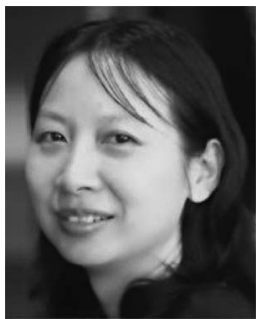

Yingying Chen received the $\mathrm{PhD}$ degree in computer science from Rutgers University. She is an Associate Professor in the Department of Electrical and Computer Engineering at Stevens Institute of Technology. Her research interests include cyber security and privacy, mobile computing, mobile healthcare, and wireless networks. She has published extensively in these areas in both journal articles and referred conference papers. Prior to joining Stevens, she was with Alcatel-Lucent. Dr. Chen is the recipient of the NSF CAREER Award and Google Research Award. She also received NJ Inventors Hall of Fame Innovator Award. She is the recipient of the Best Paper Award from ACM International Conference on Mobile Computing and Networking (MobiCom) 2011. She also received the IEEE Outstanding Contribution Award from IEEE New Jersey Coast Section each year 2005-2009. Her research has been reported in numerous media outlets.

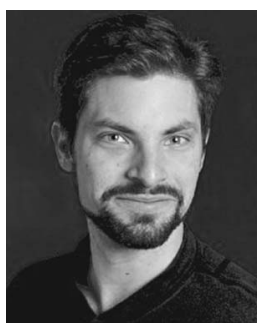

Richard P. Martin received the BA degree from Rutgers University and the MS and PhD degrees in computer science at the University of California at Berkeley. He is an Associate Professor of computer science at Rutgers University and a member of the Rutgers Wireless Network Information Laboratory (WINLAB). His current research interests include parallel computing, wireless device localization, and human factors in dependable computing. His awards include the best paper award at the IEEE Conference on Sensor and Ad Hoc Communication Networks and the ACM conference on Mobile Computing and Networking, as well as a CAREER award from the National Science Foundation. Dr. Martin has served as an investigator on grants from the Defense Advanced Research Projects Agency, the National Science Foundation, and IBM.

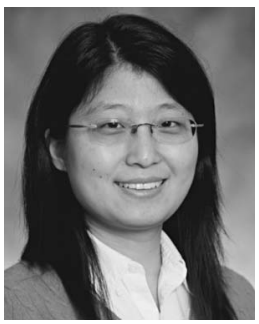

Xiaoyan Li received the $\mathrm{PhD}$ degree in computer science from Rutgers, the State University of New Jersey, in 2006. She is currently associate professor in the Department of Computer Science at Lafayette College. Her research interests include wireless networking, sensor networks, mobile computing, and distributed systems.

$\triangleright$ For more information on this or any other computing topic, please visit our Digital Library at www.computer.org/publications/dlib. 